\title{
Oral Appliances in the Management of Obstructive Sleep Apnea
}

Jing Hao Ng, BDS (Singapore), MDS Orthodontics (Singapore), MOrth RCS (Edinburgh, UK)* , Mimi Yow, BDS (Singapore), FDS RCS (Edinburgh), MSc (London) (Orthodontics), FAMS (Craniofacial Orthodontics)

\section{KEYWORDS}

- OSA • Oral appliance • Mandibular advancement • Tongue stabilizer

\section{KEY POINTS}

- The concept in oral appliances for obstructive sleep apnea (OSA) management is protrusion of the mandible and/or tongue for structural effects on the upper airway.

- The upper airway is a muscular tube and its dimensions are enlarged with mandibular and tongue advancement.

- Protrusion of the mandible and tongue stretches the muscles, thereby reducing upper airway collapsibility with airway shape change and increase in muscle tone.

- Oral appliances are effective and evidence-based options in managing OSA.

\section{TYPES OF ORAL APPLIANCES}

The primary oral appliance $(\mathrm{OA})$ used in obstructive sleep apnea (OSA) treatment is the mandibular advancement device (MAD). MADs may be either an over-the-counter stock device or customized for individual patients. MADs come in various designs and materials, but most comprise upper and lower splints mounted over the dentition as either a 1-piece monoblock (Fig. 1) or a 2-piece biblock (Fig. 2). Connectors or blocks relate the upper and lower splints in a biblock to protrude the mandible in a forward position during sleep.

Tongue-retaining devices, or tongue-stabilizing devices (TSDs) (Fig. 3), are a second type of OA, which displace the tongue anteriorly and may be customized or come in different stock sizes. TSDs use negative pressure and salivary adhesion to hold onto the tongue and anterior lip shields to elongate and reposition the tongue in a more forward position independent of the mandible during sleep, thereby opening the oropharyngeal airway. ${ }^{1,2}$

TSDs have similar efficacy as MADs but poorer compliance. More than $90 \%$ of patients preferred MADs over TSDs for OA therapy. ${ }^{3}$ The evidence base is stronger for MADs and considerably lower for TSDs. ${ }^{2,4,5}$

\section{ORAL APPLIANCE EFFECTS ON AIRWAY Cross-Sectional Area}

Airway imaging with cone-beam computed tomography, magnetic resonance imaging and nasal endoscopy showed anteroposterior (AP) mandibular protrusion predominantly increases the caliber of the airway at the retropalatal area via lateral expansion and displacement of parapharyngeal fat pads ${ }^{2,6-13}$ while the tongue and tongue-base muscles shift forward. ${ }^{2,9,13}$

The lateral widening from AP movement is attributed to stretching of soft tissue connections

Disclosure Statement: The authors declare no conflicts of interest and no funding was received in the preparation of this article.

Department of Orthodontics, National Dental Centre Singapore, 5 Second Hospital Avenue, Singapore 168938, Singapore

* Corresponding author.

E-mail address: ng.jing.hao@singhealth.com.sg 


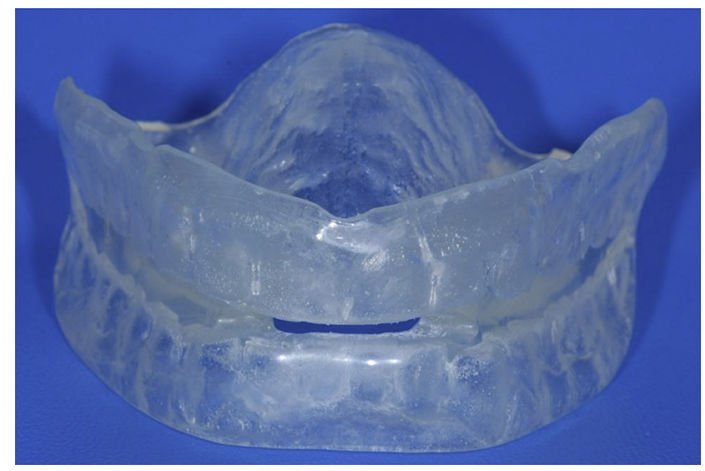

Fig. 1. Monoblock of 1-piece MAD. (Courtesy of the Orthodontic Laboratory in the National Dental Centre of Singapore, Singapore.)

between the tongue, soft palate, and lateral pharyngeal walls. ${ }^{11,14,15}$ Dynamic MRI suggests a direct connection between lateral pharyngeal walls and the ramus, postulated to be the pterygomandibular raphe. ${ }^{13}$

TSDs increase airway AP diameter to a greater degree than MADs, and traction on intrapharyngeal connections through the tongue base additionally increases the lateral dimension of the airway. Compared with MADs, TSDs produce greater increases in retropalatal and retroglossal crosssectional area (CSA). This is attributed to greater anterior tongue movement with TSDs. ${ }^{2}$

\section{Other Effects}

Lateral expansion with both MADs and TSDs promotes an elliptical cross-sectional shape with a transverse long axis. ${ }^{2}$ A small but significant decrease in upper airway length has been found with MAD use, ${ }^{2}$ which may counteract the airway length increase from lying supine demonstrated in OSA patients. ${ }^{16}$

Electromyography shows that MADs increase activation of masseter, lateral pterygoid, genioglossus, and geniohyoid muscles. It is postulated that on top of the purely structural anatomic effects of MADs, increased neuromuscular activation contributes to upper airway patency. ${ }^{17-19}$

\section{Collapsibility}

Morphologic increase in CSA, change in crosssectional shape, decrease in airway length, and neuromuscular activation with MAD may contribute to reduced collapsibility. ${ }^{2,12,20}$ Empirically, a significant reduction in upper airway collapsibility in stage 2 and stage 3 sleep was observed with MAD use. Improvement in collapsibility was significantly greater in complete responders than in partial responders or nonresponders. ${ }^{21}$ Airway collapsibility may have a dose-dependent relationship with mandibular advancement. ${ }^{10}$

\section{ORAL APPLIANCE EFFECTS ON OBSTRUCTIVE SLEEP APNEA COMPARED WITH CONTINUOUS POSITIVE AIRWAY PRESSURE}

Continuous positive airway pressure (CPAP) and MADs are chronic, noninvasive, symptomatic treatments for OSA that do not treat the underlying

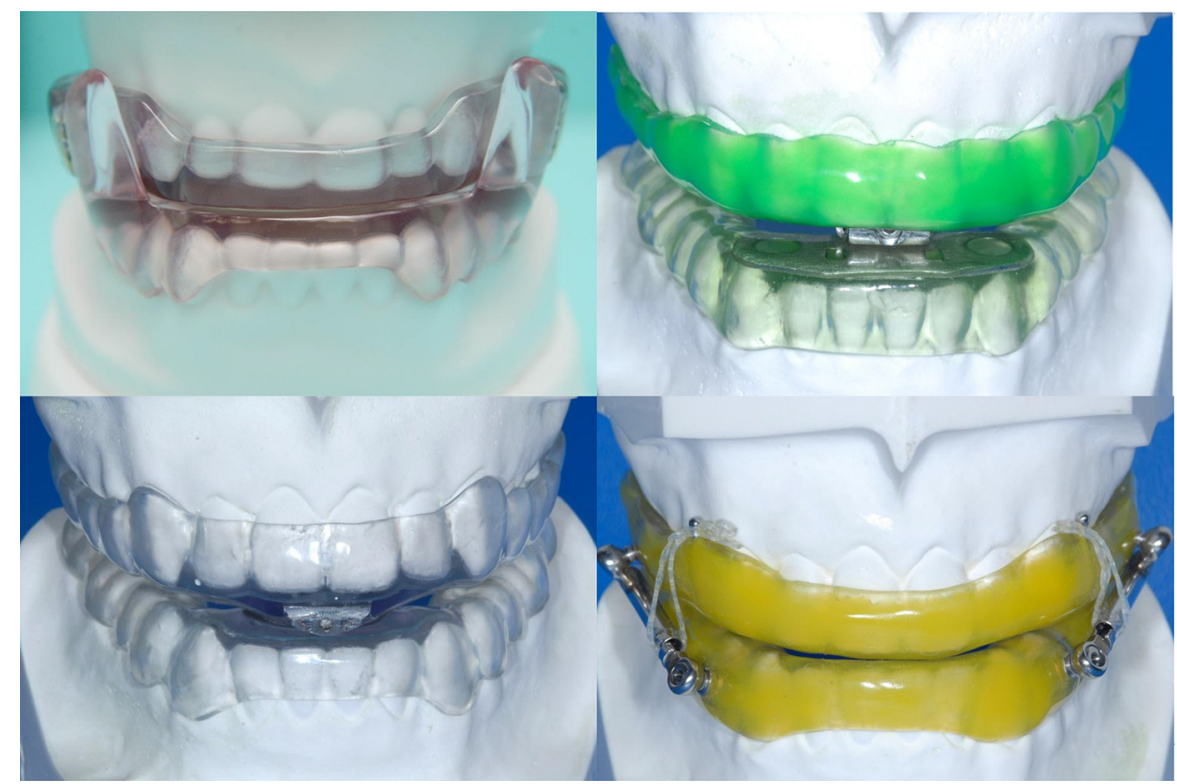

Fig. 2. Biblock or 2-piece MADs with different connectors. (Courtesy of [upper left] SomnoMed, Sydney, Australia; and [upper right, lower left, and lower right] Orthodontic Master, Singapore.) 
Fig. 3. TSD. (Courtesy of Innovative Health Technologies Ltd, Dunedin, New Zealand.)

anatomic basis of the condition. Pneumatic splinting of the airway with CPAP is acknowledged as the gold standard for treatment of OSA, although MAD has been progressively recommended with each iteration of the American Academy of Sleep Medicine (AASM) guidelines. ${ }^{4,22,23}$ The latest AASM guidelines do not specify a particular disease severity for MAD use due to lack of evidence relating MAD efficacy to disease severity. ${ }^{4}$

\section{Apnea-Hypopnea Index}

MADs are efficacious in reducing apneahypopnea index (AHI) although there is individual variability. ${ }^{24}$ A nonlinear dose-dependent relationship with degree of advancement is reported. ${ }^{25-30}$

The efficacy of CPAP over MAD in reducing $\mathrm{AHI}$ is well established and has been consistently reported by meta-analyses comparing the 2,4,31-34 even with increasing numbers of primary studies. According to the AASM/American Academy of Dental Sleep Medicine (AADSM) task force, CPAP is significantly better than MAD at reducing AHI scores (difference of 6.24 events per hour). ${ }^{4} \mathrm{~A}$ recent meta-analysis of 14 randomized controlled trials reported that CPAP reduced $\mathrm{AHI}$ by an additional 8.43 events per hour over MAD. ${ }^{34}$

\section{Oxygenation}

MADs are efficacious in improving minimum arterial oxygen saturation $\left(\mathrm{SaO}_{2}\right)$, and the improvement has been reported to have a nonlinear dose-dependent relationship with degree of advancement. ${ }^{10}$

Similar to AHI, the finding of better improvement in $\mathrm{SaO}_{2}$ with CPAP over MAD has been consis-

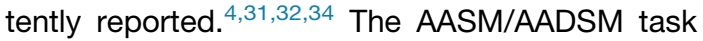
force reported that CPAP is slightly better at improving oxygenation $\left(\mathrm{SaO}_{2}\right.$ difference of $3.11 \%)$ compared with MADs. ${ }^{4}$ More recently, a significant effect on $\mathrm{SaO}_{2}$ in favor of CPAP was reported. ${ }^{34}$

\section{Compliance}

Comparing subjective self-reported MAD compliance with objective recorded CPAP compliance, MAD compliance is better. The AASM/AADSM task force reported that MADs were used 0.70 more hours per night than CPAP. ${ }^{4}$ Objective measurements of OA usage after 3 months of OA treatment ranged from a mean of 6.4 hours to 6.6 hours per night. ${ }^{35,36}$ A meta-analysis of 6 studies encompassing both subjective and objective MAD compliance measures reported an additional 1.1 hours per night use of MAD over CPAP. ${ }^{33}$

A majority of crossover trials report that MADs are preferred to CPAP, which may imply better patient compliance with MADs. ${ }^{4,37,38}$ Depending on OSA severity and total sleep time, less than ideal compliance with CPAP can substantially decrease its effectiveness. Better MAD compliance over CPAP could be why health outcomes of the 2 treatment modalities are similar despite better CPAP efficacy at reducing $\mathrm{AHI}$ and increasing oxygenation. ${ }^{37}$

\section{Subjective Sleepiness}

MADs improve subjective daytime sleepiness measured by Epworth Sleepiness Scale (ESS) scores significantly. ${ }^{39}$

Despite better efficacy with CPAP than MADs in reducing $\mathrm{AHI}$ and improving $\mathrm{SaO}_{2}$, differences in ESS scores are more ambiguous. A network meta-analysis of 67 studies found that CPAP and MADs are both effective in reducing excessive daytime sleepiness as assessed by the ESS, but CPAP is likely to be more effective than MADs, with a greater reduction in ESS by an average of 0.8 points. ${ }^{40}$ Other reviews have reported either similar outcomes ${ }^{4,32}$ or slightly better outcomes with CPAP. ${ }^{33,34}$

\section{Function and Quality of Life}

Aside from long-term health effects, untreated OSA also affects health-related quality of life, with hypersomnolence impacting ability to function. ${ }^{41}$ Function and quality of life are commonly measured using both the sleep-specific Functional Outcomes of Sleep Questionnaire (FOSQ) and generic Medical Outcomes Study 36-Item Short Form Health Survey (SF-36). MADs are associated with improvements in FOSQ subscale and total scores as well as SF-36 scores. ${ }^{4,42,43}$

Minimal difference was found between CPAP and MAD with respect to improvements in daytime functional outcomes measured by SF-36 and FOSQ scores. ${ }^{4}$ This finding was corroborated in a meta-analysis, ${ }^{44}$ with direct comparisons as well 
as network meta-analysis showing no significant difference in treatment outcomes between CPAP and MAD in the mental and physical components of SF-36. Other meta-analyses have also reported no difference in FOSQ or SF-36 scores. ${ }^{31-34}$

\section{Cardiovascular Effects}

Meta-analyses by multiple groups have found MADs to be the equivalent of CPAP at reducing blood pressure in adults with OSA. ${ }^{4,34,45-47} \mathrm{~A}$ network meta-analysis reported that CPAP was associated with a reduction in systolic blood pressure of $2.5 \mathrm{~mm} \mathrm{Hg}$ and diastolic blood pressure reduction of $2.0 \mathrm{~mm} \mathrm{Hg}$. MADs were associated with reduction in systolic blood pressure of $2.1 \mathrm{~mm} \mathrm{Hg}$ and diastolic blood pressure of $1.9 \mathrm{~mm} \mathrm{Hg}$. There was no significant difference between CPAP and MADs. ${ }^{40}$

Studies on other cardiovascular markers (heart rate variability, circulating cardiovascular biomarkers, endothelial function, and arterial stiffness) were deemed heterogeneous and inconclusive. ${ }^{46}$

\section{Mortality}

A prospective cohort study on long-term cardiovascular mortality in 208 subjects found CPAP and MAD equally effective in reducing the risk of fatal cardiovascular events in patients with severe OSA, despite higher residual $A H I$ for MAD compared with CPAP users (16.3 vs 4.5 events per hour). There was no difference in the cumulative cardiovascular mortality between OSA patients treated with CPAP or MAD and that of the nonapneic controls, suggesting effective symptomatic treatment with either modality despite differences in residual $\mathrm{AHI} .{ }^{48}$ This outcome, however, is from a single observational study. More longitudinal studies with better methodology are needed to answer key questions on cardiovascular morbidity and mortality outcomes. ${ }^{46,48}$

\section{ORAL APPLIANCE ADVERSE EFFECTS}

Similar to efficacy, adverse side effects have a dose-dependent relationship with protrusion. ${ }^{29} \mathrm{~A}$ balance must be struck between efficacy and side effects because more adverse effects reduce long-term compliance, ${ }^{35,49}$ resulting in patients terminating MAD therapy. ${ }^{50,51}$ Most adverse effects caused by MADs are mild and transient, occur during the initial phase of therapy, and tend to resolve with time. ${ }^{51,52}$

\section{Dental Effects}

Long-term use of OAs leads to occlusal changes. A meta-analysis reported a significant increase in lower incisor inclination by $2.07^{\circ}$, resulting in $0.99-\mathrm{mm}$ decrease in overjet (OJ) and $1.0-\mathrm{mm}$ decrease in overbite (OB). A greater decrease in $\mathrm{OJ}$ and $\mathrm{OB}$ was associated with longer treatment. There was no significant change in the upper incisor inclination or interincisal angle. ${ }^{53}$

Due to the lower incisor inclination change and attendant decrease in OJ and OB, there was significant increase in anterior crossbites, ${ }^{54}$ increase in mandibular arch width, and reduction in lower arch crowding. ${ }^{54,55}$ Maxillary arch width increase and upper arch decrowding also have been reported. ${ }^{55} \mathrm{~A}$ decrease in posterior occlusal contacts ${ }^{54,56-58}$ may result in a transient difficulty with chewing. ${ }^{51}$ Although the magnitude of dental changes are small, they may be significant to patient perception of developing dental malocclusion. ${ }^{59}$

\section{Craniofacial Changes}

A meta-analysis of skeletal changes from MAD use reported no significant skeletal changes. ${ }^{53}$ Other investigators, however, report significant increase in lower and total anterior facial height from longterm MAD use of more than 2 years. ${ }^{60,61}$

\section{Temporomandibular Joint Disorders}

Transient muscle soreness and temporomandibular joint (TMJ) discomfort have been reported after MAD use, especially during the initial titration period. ${ }^{3,51}$ A 5-year follow-up study of MAD patients found mild, temporary subjective side effects, such as muscular or TMJ discomfort, but no changes in temporomandibular disorder prevalence using the Research Diagnostic Criteria for Temporomandibular Disorders. ${ }^{57}$

\section{Other Effects}

Other MAD side effects include increased salivation, more frequent and excessive dry mouth, tongue discomfort, and a sense of suffocation. ${ }^{3,35,51}$ TSD side effects include excess salivation, drooling, dry mouth, and soft tissue irritation. ${ }^{3}$

\section{PREDICTING AND IMPROVING ORAL APPLIANCE TREATMENT RESPONSE}

Although most patients show an increased airway CSA with mandibular advancement, a minority of patients show no change or even a decreased CSA. ${ }^{9,12}$ MAD is a primarily structural treatment ${ }^{9}$ for a heterogeneous condition with nonanatomic etiology in up to $56 \%$ of patients. ${ }^{62,63}$ Consequently, MAD treatment completely resolves $\mathrm{AHI}$ to fewer than 5 events per hour in only $36 \%$ to $70 \%$ of OSA patients. ${ }^{24,38}$ Patients of female gender, younger age, smaller neck circumference, lower body mass 
index, lower AHI, and supine-dependent OSA are predicted to have better treatment success with MADs. ${ }^{38,50,64,65}$ For TSDs, the best predictor of success is believed to be the presence of a single-site airway obstruction in supine-dependent OSA. ${ }^{1}$

There is currently no validated clinical method to reliably differentiate responders from nonresponders. ${ }^{24}$ Uncertainty of MAD treatment response is exacerbated by cost of treatment. ${ }^{66}$ The literature lacks consensus in successful treatment outcome, with a majority of success criteria used in research not meshing with clinical definitions of OSA severity. Discordant success criteria makes comparing results of different studies difficult. ${ }^{67}$ Prospective trials are under way to determine predictive potential of wakeful nasoendoscopy with Müller maneuver, drug-induced sleep endoscopy (DISE), and computational fluid dynamics. ${ }^{68,69}$ In the meantime, careful diagnosis and patient selection can increase the chances of success.

\section{Cephalometry}

Classically, shorter soft palate length, larger retropalatal airway space, lower hyoid bone position, and a smaller mandible are associated with favorable MAD treatment response. ${ }^{1,65}$ Two recent systematic reviews, ${ }^{70,71}$ however, exploring cephalometric predictors for MAD response found a majority of observational studies on cephalometric predictors had flawed designs and failed to control for known confounding factors such as age, gender, body mass index, and baseline AHI. Definitions of treatment success were inconsistent, and heterogeneity in design prevented data synthesis and meta-analysis. Cephalometric parameters warranting further study included mandibular plane angle, hyoid to mandibular plane distance, and soft palate length.

\section{Mandibular Advancement Device Design}

\section{Customized versus noncustomized}

Both customized and noncustomized MADs reduce $\mathrm{AHI}$ in adult patients with OSA, but meta-analysis by the AASM/AADSM task force shows the improvements to be far greater in customized than noncustomized, with an $\mathrm{AHI}$ reduction of 13.89 events compared with 6.28 events per hour. ${ }^{4}$ Noncustomized MADs do not improve minimum $\mathrm{SaO}_{2}$, whereas customized MADs increase $\mathrm{SaO}_{2}$ by $3.22 \% .{ }^{4}$ They also do not reduce ESS scores to any significant level, ${ }^{72}$ whereas modest improvements in ESS scores can be expected from customized MADs. ${ }^{4}$

\section{Titratable versus nontitratable}

Both custom titratable and custom nontitratable MADs reduce $\mathrm{AHI}$, improve $\mathrm{SaO}_{2}$ and reduce
ESS scores, with meta-analyses by the AASM/ AADSM task force showing the improvements to be approximately equivalent. Titratable MADs are recommended, however, over nontitratable MADs because the confidence interval for the effect of custom, titratable MADs is considerably smaller than for custom, nontitratable MADs. ${ }^{4}$

Due to dose-dependent effects, a clinical titration can improve MAD response and increase the amount of achievable protrusion. This is only possible with titratable MADs. ${ }^{27}$

\section{Therapeutic Diagnosis}

\section{Visualizing airway response}

Treatment response to MAD can be estimated by visualizing airway response to mandibular protrusion or tongue thrust with nasoendoscopy or MRI in awake supine patients, or in patients using DISE. An increase in velopharyngeal CSA with mandibular advancement was significantly associated with greater $\mathrm{AHI}$ reduction with MADs. , $^{8,73,74}$ This was not fully predictive, however, with some patients without velopharyngeal widening showing high $\mathrm{AHI}$ reductions and vice versa. ${ }^{2,73}$

Müller maneuver during nasoendoscopy induced significantly greater collapse in velopharyngeal and oropharyngeal CSA in MAD nonresponders than responders. With mandibular advancement, Müller maneuver induced a significantly greater collapse at all airway levels in nonresponders. ${ }^{8}$

On MRI, TSD responders showed a greater increase with TSD in AP diameter, minimum and mean CSA, and volume compared with nonresponders. ${ }^{2}$

\section{Mandibular advancement device titration using polysomnography}

Due to the dose-dependent relationship between efficacy and protrusion, increasing protrusion can increase treatment response in OSA patients. Conventional titration protocols use subjective symptoms to adjust mandibular advancement over several months and an outcome polysomnography (PSG) to confirm efficacy. There is no consensus, however, on an optimal titration protocol. ${ }^{67}$

Manually increasing MAD advancement during overnight PSG can increase therapeutic efficacy of mandibular protrusion while reducing the number of titration visits required by most therapeutic protocols. After a clinical titration period, overnight MAD titration with PSG using the final MAD increased treatment response by an additional $9.9 \%$ to $30.4 \%$ of study subjects. ${ }^{28,75}$ Disappointing outcomes were reported, however, using an interim MAD without a period of clinical titration 
because efficacy of the interim MAD was not translated to the final MAD. ${ }^{76}$

Overnight MAD titration with PSG may require large single-night mandibular advancements to relieve respiratory events, in contrast to slow titration with conventional protocols. Significant jaw discomfort was noted with PSG titration without prior MAD use ${ }^{76}$ but was not reported by patients who had an adaptation period with conventional clinical MAD titration. ${ }^{28}$

\section{Remote-controlled mandibular positioners}

Using remote-controlled mandibular positioners (RCMPs) for single-night titration of MADs is similar to overnight MAD titration with PSG, with the advantage of adjustment done remotely without waking the patient or removing the appliance. Sleep architecture is maintained while protruding the mandible progressively until respiratory events are eliminated.

RCMPs can determine an effective target protrusion that is correlated with successful MAD treatment, may be able to identify nonresponders early, and reduce the number of titration reviews needed compared with conventional titration protocols. ${ }^{81}$ RCMP titration, however, necessitates large single-night mandibular advancements, which can cause significant jaw discomfort. ${ }^{26}$

\section{Combination Therapy}

Mandibular advancement device and tonguestabilizing devices

Because TSDs have somewhat different anatomic effects on the airway compared with MADs, ${ }^{2}$ combining the therapies using a novel hybrid appliance resulted in augmentative treatment effects. Tongue suction with $6 \mathrm{~mm}$ mandibular protrusion produced better treatment response than $8 \mathrm{~mm}$ mandibular protrusion alone. ${ }^{30}$ Combination therapy might improve OA treatment response and prove useful in patients with limited mandibular protrusion.

\section{Mandibular advancement device and continuous positive airway pressure}

Combining MAD and CPAP lowered the therapeutic CPAP pressure. ${ }^{82-85}$ An augmentative treatment effect on reducing $\mathrm{AHI}$ was found in patients not responding to CPAP or MAD use alone. $^{82-84}$

\section{PRACTICE RECOMMENDATIONS Indications}

OAs in the form of MADs can be used as an alternative for patients of all OSA severities who are intolerant of CPAP or prefer an alternative therapy. ${ }^{4}$

\section{Contraindications}

Patients who are generally unsuitable for MAD treatment include edentulous patients and patients with inadequate number of sound teeth, severe periodontitis, and/or history of TMJ disease. There are exceptions, and MADs can sometimes be worn successfully by edentulous patients with good dentoalveolar ridges. ${ }^{1}$ TSDs are not dependent on the dentition for retention and can be prescribed for edentulous or insufficiently dentate patients.

\section{Appliance Selection}

A plethora of designs is currently in use. ${ }^{86}$ Based on current evidence, customized, titratable MADs are the preferred form of OAs. ${ }^{4}$ TSDs can be used in MAD nonresponders who want OA therapy.

\section{Clinical Follow-up}

Trained dentists in sleep practice should follow-up patients using MADs to reduce dental side effects and occlusal changes. ${ }^{4}$ Initial side effects are transient and reversible. ${ }^{51,52}$ Close monitoring and coaching through the initial period may improve compliance. ${ }^{35,50,51}$

For initial comfort, mandibular advancement is $75 \%$ of maximum protrusion. ${ }^{1}$ Clinical titration with an adaptation period increases the achievable protrusion and improves treatment response. ${ }^{27}$

Clinical titration should be followed by overnight PSG. Subjective feedback is not sufficient to determine the optimal setting of MAD. Post-PSG titration has been shown to improve MAD efficacy significantly. ${ }^{4,28,75}$

\section{REFERENCES}

1. Yow M, Lye EKW. Obstructive sleep apnea: orthodontic startegies to establish and maintain a patent airway. In: Krishnan V, Davidovitch Z, editors. Integrated clinical orthodontics. 1st edition. New Jersey (USA): Wiley-Blackwell Publishing Ltd; 2012. p. 214-39.

2. Sutherland K, Deane SA, Chan AS, et al. Comparative effects of two oral appliances on upper airway structure in obstructive sleep apnea. Sleep 2011; 34(4):469-77.

3. Deane SA, Cistulli PA, Ng AT, et al. Comparison of mandibular advancement splint and tongue stabilizing device in obstructive sleep apnea: a randomized controlled trial. Sleep 2009;32(5):648-53.

4. Ramar K, Dort LC, Katz SG, et al. Clinical practice guideline for the treatment of obstructive sleep apnea and snoring with oral appliance therapy: an update for 2015. J Clin Sleep Med 2015;11(7): 773-827. 
5. Chang ET, Fernandez-Salvador C, Giambo J, et al. Tongue retaining devices for obstructive sleep apnea: a systematic review and meta-analysis. Am J Otolaryngol 2017;38(3):272-8.

6. Ishida M, Inoue $Y$, Suto $Y$, et al. Mechanism of action and therapeutic indication of prosthetic mandibular advancement in obstructive sleep apnea syndrome. Psychiatry Clin Neurosci 1998;52(2):227-9.

7. Ryan CF, Love LL, Peat D, et al. Mandibular advancement oral appliance therapy for obstructive sleep apnoea: effect on awake calibre of the velopharynx. Thorax 1999;54(11):972-7.

8. Chan AS, Lee RW, Srinivasan VK, et al. Nasopharyngoscopic evaluation of oral appliance therapy for obstructive sleep apnoea. Eur Respir J 2010; 35(4):836-42.

9. Chan AS, Sutherland K, Schwab RJ, et al. The effect of mandibular advancement on upper airway structure in obstructive sleep apnoea. Thorax 2010; 65(8):726-32.

10. Kato J, Isono S, Tanaka A, et al. Dose-dependent effects of mandibular advancement on pharyngeal mechanics and nocturnal oxygenation in patients with sleep-disordered breathing. Chest 2000; 117(4):1065-72.

11. Kuna ST, Woodson LC, Solanki DR, et al. Effect of progressive mandibular advancement on pharyngeal airway size in anesthetized adults. Anesthesiology 2008;109(4):605-12.

12. Choi JK, Hur YK, Lee JM, et al. Effects of mandibular advancement on upper airway dimension and collapsibility in patients with obstructive sleep apnea using dynamic upper airway imaging during sleep. Oral Surg Oral Med Oral Pathol Oral Radiol Endod 2010;109(5):712-9.

13. Brown EC, Cheng S, McKenzie DK, et al. Tongue and lateral upper airway movement with mandibular advancement. Sleep 2013;36(3):397-404.

14. Isono S, Tanaka A, Sho Y, et al. Advancement of the mandible improves velopharyngeal airway patency. J Appl Physiol (1985) 1995;79(6):2132-8.

15. Isono S, Tanaka A, Tagaito $Y$, et al. Pharyngeal patency in response to advancement of the mandible in obese anesthetized persons. Anesthesiology 1997;87(5):1055-62.

16. Pae EK, Lowe AA, Fleetham JA. A role of pharyngeal length in obstructive sleep apnea patients. Am J Orthod Dentofacial Orthop 1997;111(1):12-7.

17. Yoshida K. Effect of a prosthetic appliance for treatment of sleep apnea syndrome on masticatory and tongue muscle activity. J Prosthet Dent 1998;79(5): 537-44.

18. Johal A, Gill G, Ferman A, et al. The effect of mandibular advancement appliances on awake upper airway and masticatory muscle activity in patients with obstructive sleep apnoea. Clin Physiol Funct Imaging 2007;27(1):47-53.
19. Kurtulmus H, Cotert S, Bilgen C, et al. The effect of a mandibular advancement splint on electromyographic activity of the submental and masseter muscles in patients with obstructive sleep apnea. Int J Prosthodont 2009;22(6):586-93.

20. Malhotra A, Huang Y, Fogel RB, et al. The male predisposition to pharyngeal collapse: importance of airway length. Am J Respir Crit Care Med 2002; 166(10):1388-95.

21. Ng AT, Gotsopoulos H, Qian J, et al. Effect of oral appliance therapy on upper airway collapsibility in obstructive sleep apnea. Am J Respir Crit Care Med 2003;168(2):238-41.

22. Kushida CA, Morgenthaler TI, Littner MR, et al. Practice parameters for the treatment of snoring and Obstructive Sleep Apnea with oral appliances: an update for 2005. Sleep 2006;29(2):240-3.

23. Practice parameters for the treatment of snoring and obstructive sleep apnea with oral appliances. American Sleep Disorders Association. Sleep 1995;18(6): 511-3.

24. Sutherland K, Vanderveken OM, Tsuda H, et al. Oral appliance treatment for obstructive sleep apnea: an update. J Clin Sleep Med 2014;10(2):215-27.

25. Raphaelson MA, Alpher EJ, Bakker KW, et al. Oral appliance therapy for obstructive sleep apnea syndrome: progressive mandibular advancement during polysomnography. Cranio 1998;16(1):44-50.

26. Tsai WH, Vazquez JC, Oshima T, et al. Remotely controlled mandibular positioner predicts efficacy of oral appliances in sleep apnea. Am J Respir Crit Care Med 2004;170(4):366-70.

27. Gindre L, Gagnadoux F, Meslier N, et al. Mandibular advancement for obstructive sleep apnea: dose effect on apnea, long-term use and tolerance. Respiration 2008;76(4):386-92.

28. Almeida FR, Parker JA, Hodges JS, et al. Effect of a titration polysomnogram on treatment success with a mandibular repositioning appliance. J Clin Sleep Med 2009;5(3):198-204.

29. Aarab G, Lobbezoo F, Hamburger HL, et al. Effects of an oral appliance with different mandibular protrusion positions at a constant vertical dimension on obstructive sleep apnea. Clin Oral Investig 2010;14(3):339-45.

30. Dort L, Remmers J. A combination appliance for obstructive sleep apnea: the effectiveness of mandibular advancement and tongue retention. J Clin Sleep Med 2012;8(3):265-9.

31. Giles TL, Lasserson TJ, Smith BH, et al. Continuous positive airways pressure for obstructive sleep apnoea in adults. Cochrane Database Syst Rev 2006;(3):CD001106.

32. Lim J, Lasserson TJ, Fleetham J, et al. Oral appliances for obstructive sleep apnoea. Cochrane Database Syst Rev 2006;(1):CD004435.

33. Schwartz M, Acosta L, Hung YL, et al. Effects of CPAP and mandibular advancement device 
treatment in obstructive sleep apnea patients: a systematic review and meta-analysis. Sleep Breath 2018;22(3):555-68.

34. Zhang M, Liu Y, Liu Y, et al. Effectiveness of oral appliances versus continuous positive airway pressure in treatment of OSA patients: an updated meta-analysis. Cranio 2018;1-18.

35. Dieltjens M, Verbruggen AE, Braem MJ, et al. Determinants of objective compliance during oral appliance therapy in patients with sleep-disordered breathing: a prospective clinical trial. JAMA Otolaryngol Head Neck Surg 2015;141(10):894-900.

36. Vanderveken OM, Dieltjens $M$, Wouters $\mathrm{K}$, et al. Objective measurement of compliance during oral appliance therapy for sleep-disordered breathing. Thorax 2013;68(1):91-6.

37. Sutherland K, Phillips CL, Cistulli PA. Efficacy versus effectiveness in the treatment of obstructive sleep apnea: CPAP and oral appliances. J Dent Sleep Med 2015;2(4):175-81.

38. Lettieri CJ, Almeida FR, Cistulli PA, et al. Oral appliances for the treatment of obstructive sleep apneahypopnea syndrome and for concomitant sleep bruxism. In: $\mathrm{MH} \mathrm{K}, \mathrm{T} R, \mathrm{WC} \mathrm{D}$, editors. Principles and practice of sleep medicine. 6th edition. Philadelphia: Elsevier; 2017. p. 1445-57.

39. Ahrens A, McGrath C, Hagg U. Subjective efficacy of oral appliance design features in the management of obstructive sleep apnea: a systematic review. Am J Orthod Dentofacial Orthop 2010;138(5):559-76.

40. Bratton DJ, Gaisl T, Schlatzer C, et al. Comparison of the effects of continuous positive airway pressure and mandibular advancement devices on sleepiness in patients with obstructive sleep apnoea: a network meta-analysis. Lancet Respir Med 2015;3(11):869-78.

41. Ng A, Gotsopoulos H, Darendeliler AM, et al. Oral appliance therapy for obstructive sleep apnea. Treat Respir Med 2005;4(6):409-22.

42. Blanco J, Zamarron C, Abeleira Pazos MT, et al. Prospective evaluation of an oral appliance in the treatment of obstructive sleep apnea syndrome. Sleep Breath 2005;9(1):20-5.

43. Gauthier L, Laberge L, Beaudry M, et al. Efficacy of two mandibular advancement appliances in the management of snoring and mild-moderate sleep apnea: a cross-over randomized study. Sleep Med 2009; 10(3):329-36.

44. Kuhn E, Schwarz El, Bratton DJ, et al. Effects of CPAP and mandibular advancement devices on health-related quality of life in OSA: a systematic review and meta-analysis. Chest 2017;151(4):786-94.

45. Bratton DJ, GaisI T, Wons AM, et al. CPAP vs mandibular advancement devices and blood pressure in patients with obstructive sleep apnea: a systematic review and meta-analysis. Jama 2015;314(21):2280-93.

46. de Vries GE, Wijkstra PJ, Houwerzijl EJ, et al. Cardiovascular effects of oral appliance therapy in obstructive sleep apnea: a systematic review and meta-analysis. Sleep Med Rev 2018;40:55-68.

47. Van Haesendonck G, Dieltjens M, Kastoer C, et al. Cardiovascular benefits of oral appliance therapy in obstructive sleep apnea: a systematic review. J Dent Sleep Med 2015;2(1):9-14.

48. Anandam A, Patil M, Akinnusi M, et al. Cardiovascular mortality in obstructive sleep apnoea treated with continuous positive airway pressure or oral appliance: an observational study. Respirology 2013; 18(8):1184-90.

49. Attali V, Chaumereuil C, Arnulf I, et al. Predictors of long-term effectiveness to mandibular repositioning device treatment in obstructive sleep apnea patients after 1000 days. Sleep Med 2016;27-28:107-14.

50. Marklund M, Stenlund H, Franklin KA. Mandibular advancement devices in 630 men and women with obstructive sleep apnea and snoring: tolerability and predictors of treatment success. Chest 2004; 125(4):1270-8.

51. de Almeida FR, Lowe AA, Tsuiki S, et al. Long-term compliance and side effects of oral appliances used for the treatment of snoring and obstructive sleep apnea syndrome. J Clin Sleep Med 2005;1(2):143-52.

52. Ferguson KA, Cartwright R, Rogers R, et al. Oral appliances for snoring and obstructive sleep apnea: a review. Sleep 2006;29(2):244-62.

53. Araie $\mathrm{T}$, Okuno K, Ono Minagi $\mathrm{H}$, et al. Dental and skeletal changes associated with long-term oral appliance use for obstructive sleep apnea: a systematic review and meta-analysis. Sleep Med Rev 2018;41:161-72.

54. Pliska BT, Nam $H$, Chen $H$, et al. Obstructive sleep apnea and mandibular advancement splints: occlusal effects and progression of changes associated with a decade of treatment. J Clin Sleep Med 2014; 10(12):1285-91.

55. Chen H, Lowe AA, de Almeida FR, et al. Threedimensional computer-assisted study model analysis of long-term oral-appliance wear. Part 2. Side effects of oral appliances in obstructive sleep apnea patients. Am J Orthod Dentofacial Orthop 2008; 134(3):408-17.

56. Otsuka R, Almeida FR, Lowe AA. The effects of oral appliance therapy on occlusal function in patients with obstructive sleep apnea: a short-term prospective study. Am J Orthod Dentofacial Orthop 2007; 131(2):176-83.

57. Martinez-Gomis J, Willaert E, Nogues L, et al. Five years of sleep apnea treatment with a mandibular advancement device. Side effects and technical complications. Angle Orthod 2010;80(1):30-6.

58. Fransson AMC, Kowalczyk A, Isacsson G. A prospective 10-year follow-up dental cast study of patients with obstructive sleep apnoea/snoring who use a mandibular protruding device. Eur J Orthod 2017;39(5):502-8. 
59. Alessandri-Bonetti G, D'Anto V, Stipa C, et al. Dentoskeletal effects of oral appliance wear in obstructive sleep apnoea and snoring patients. Eur J Orthod 2017;39(5):482-8.

60. Almeida FR, Lowe AA, Sung JO, et al. Long-term sequellae of oral appliance therapy in obstructive sleep apnea patients: Part 1. Cephalometric analysis. Am J Orthod Dentofacial Orthop 2006;129(2): 195-204.

61. Doff MH, Hoekema A, Pruim GJ, et al. Long-term oral-appliance therapy in obstructive sleep apnea: a cephalometric study of craniofacial changes. J Dent 2010;38(12):1010-8.

62. Eckert DJ, White DP, Jordan AS, et al. Defining phenotypic causes of obstructive sleep apnea. Identification of novel therapeutic targets. Am J Respir Crit Care Med 2013;188(8):996-1004.

63. Edwards BA, Eckert DJ, Jordan AS. Obstructive sleep apnoea pathogenesis from mild to severe: is it all the same? Respirology 2017;22(1):33-42.

64. Liu Y, Lowe AA, Fleetham JA, et al. Cephalometric and physiologic predictors of the efficacy of an adjustable oral appliance for treating obstructive sleep apnea. Am J Orthod Dentofacial Orthop 2001;120(6):639-47.

65. Okuno K, Pliska BT, Hamoda M, et al. Prediction of oral appliance treatment outcomes in obstructive sleep apnea: a systematic review. Sleep Med Rev 2016:30:25-33.

66. American Sleep Assocociation / sleep apnea oral appliances - research \& treatments | 2018. Available at: https://www.sleepassociation.org/sleep-disorders/ sleep-apnea/oral-appliance-for-sleep-apnea/. Accessed July 22, 2018.

67. Dieltjens M, Vanderveken OM, Heyning $\mathrm{PH}$, et al. Current opinions and clinical practice in the titration of oral appliances in the treatment of sleepdisordered breathing. Sleep Med Rev 2012;16(2): 177-85.

68. Verbruggen AE, Vroegop AV, Dieltjens M, et al. Predicting Therapeutic Outcome of Mandibular Advancement Device Treatment in Obstructive Sleep Apnoea (PROMAD): study design and baseline characteristics. J Dent Sleep Med 2016;3(4): 119-38.

69. National Institutes of Health, United States National Library of Medicine, ClinicalTrials.gov. 2018. Available at: https://clinicaltrials.gov/ct2/show/record/ NCT01532050. Accessed 26 July, 2018.

70. Alessandri-Bonetti G, Ippolito DR, Bartolucci ML, et al. Cephalometric predictors of treatment outcome with mandibular advancement devices in adult patients with obstructive sleep apnea: a systematic review. Korean J Orthod 2015;45(6):308-21.

71. Guarda-Nardini L, Manfredini D, Mion M, et al. Anatomically based outcome predictors of treatment for obstructive sleep apnea with intraoral splint devices: a systematic review of cephalometric studies. J Clin Sleep Med 2015;11(11):1327-34.

72. Vanderveken OM, Devolder A, Marklund M, et al. Comparison of a custom-made and a thermoplastic oral appliance for the treatment of mild sleep apnea. Am J Respir Crit Care Med 2008;178(2): 197-202.

73. Sasao Y, Nohara K, Okuno K, et al. Videoendoscopic diagnosis for predicting the response to oral appliance therapy in severe obstructive sleep apnea. Sleep Breath 2014;18(4):809-15.

74. Vroegop AV, Vanderveken OM, Dieltjens $M$, et al. Sleep endoscopy with simulation bite for prediction of oral appliance treatment outcome. J Sleep Res 2013;22(3):348-55.

75. Krishnan V, Collop NA, Scherr SC. An evaluation of a titration strategy for prescription of oral appliances for obstructive sleep apnea. Chest 2008;133(5): 1135-41.

76. Kuna ST, Giarraputo PC, Stanton DC, et al. Evaluation of an oral mandibular advancement titration appliance. Oral Surg Oral Med Oral Pathol Oral Radiol Endod 2006;101(5):593-603.

77. Petelle B, Vincent G, Gagnadoux F, et al. One-night mandibular advancement titration for obstructive sleep apnea syndrome: a pilot study. Am J Respir Crit Care Med 2002;165(8):1150-3.

78. Dort LC, Hadjuk E, Remmers JE. Mandibular advancement and obstructive sleep apnoea: a method for determining effective mandibular protrusion. Eur Respir J 2006;27(5):1003-9.

79. Remmers J, Charkhandeh S, Grosse J, et al. Remotely controlled mandibular protrusion during sleep predicts therapeutic success with oral appliances in patients with obstructive sleep apnea. Sleep 2013;36(10):1517-25, 1525A.

80. Sutherland K, Ngiam J, Cistulli PA. Performance of remotely controlled mandibular protrusion sleep studies for prediction of oral appliance treatment response. J Clin Sleep Med 2017;13(3):411-7.

81. Kastoer C, Dieltjens M, Oorts E, et al. The use of remotely controlled mandibular positioner as a predictive screening tool for mandibular advancement device therapy in patients with obstructive sleep apnea through single-night progressive titration of the mandible: a systematic review. J Clin Sleep Med 2016;12(10):1411-21.

82. Denbar MA. A case study involving the combination treatment of an oral appliance and auto-titrating CPAP unit. Sleep Breath 2002;6(3):125-8.

83. El-Solh AA, Moitheennazima B, Akinnusi ME, et al. Combined oral appliance and positive airway pressure therapy for obstructive sleep apnea: a pilot study. Sleep Breath 2011;15(2):203-8.

84. Liu HW, Chen YJ, Lai YC, et al. Combining MAD and CPAP as an effective strategy for treating patients with severe sleep apnea intolerant to high-pressure 
PAP and unresponsive to MAD. PLoS One 2017; 12(10):e0187032.

85. de Vries GE, Doff MHJ, Hoekema A, et al. Continuous positive airway pressure and oral appliance hybrid therapy in obstructive sleep apnea: patient comfort, compliance, and preference: a pilot study. J Dent Sleep Med 2016;3(1):5-10.

86. Yow M. An overview of oral appliances and managing the airway in obstructive sleep apnea. Semin Orthod 2009;15(2):88-93. 